\title{
Nonlinear Dielectric Relaxation in Solutions of the Polar Mesogenic Molecules in the Isotropic Phase
}

\author{
P. KEDZIORA \\ Institute of Molecular Physics, Polish Academy of Sciences \\ Smoluchowskiego 17, 60-179 Poznań, Poland \\ (Received April 3, 2003; in final form June 23, 2003)
}

\begin{abstract}
The nonlinear spectra of $4,4^{\prime}$ - $n$-hexylcyanobiphenyl $\left(\mathrm{C}_{6} \mathrm{H}_{13}-\mathrm{Ph}-\mathrm{Ph}-\mathrm{CN}\right)$ in benzene solutions were recorded in the frequency range of $300 \mathrm{kHz}-$ $100 \mathrm{MHz}$ in the presence of the strong static electric field $E_{0}=$ $1.1 \times 10^{7} \mathrm{~V} / \mathrm{m}$, at $25^{\circ} \mathrm{C}$. The dynamic pretransitional phenomena revealed for polar $\mathrm{C}_{6} \mathrm{H}_{13}-\mathrm{Ph}-\mathrm{Ph}-\mathrm{CN}$ were interpreted in the frame of the Landau-de Gennes theory. The identical concentration-dependent behavior of nonlinear relaxation time in the vicinity of the transition from the isotropic to the nematic phase for polar and nonpolar compounds was discussed.
\end{abstract}

PACS numbers: 64.70.Md, 72.20.Ht, 68.35.Rh

\section{Introduction}

The isotropic-nematic phase transition in liquid crystals is weakly first order. Pretransitional behavior observed in the pure isotropic mesogenic liquids in the vicinity of the transition to the nematic phase has an origin in existing of short-range orientational order among the molecules, which leads to the formation of pseudonematic domains in the isotropic phase. The size of the domains increases as the temperature of the liquid approaches to the nematic phase transition.

The Landau-de Gennes theory [1] has been successful in explaining the dynamics in isotropic liquid crystals close to the nematic phase transitions [2-10] and shows that the intermolecular correlation length $\xi$ depends on temperature in the following way:

$$
\xi(T)=\xi_{0}\left(\frac{T^{*}}{T-T^{*}}\right)^{1 / 2},
$$

where $\xi_{0}$ is of the order of the molecular length, and $T^{*}$ denotes the temperature of the virtual transition of the second order. 
The validity of the Landau-de Gennes theory has been experimentally confirmed in the measurements of the light scattering [4], the optical Kerr effect [5-9] and the transient grating optical Kerr effect [7, 8]. In our recent papers $[11,12]$ the Landau-de Gennes theory was also successfully used for interpretation of the pretransitional behavior of the nonlinear dielectric relaxation measured for pure polar nematogenic 4-(trans-4'-n-hexylcyclohexyl)isothiocyanatobenzene, $\mathrm{C}_{6} \mathrm{H}_{13}-\mathrm{CyHx}-\mathrm{Ph}-\mathrm{NCS}$ (6CHBT) [12], as well as for its solutions in nonpolar medium (benzene) [11]. For pure nematogenic 6CHBT [12] the temperature dependence of the relaxation time $\tau$ for the reorientation of pseudonematic domains in the medium of the viscosity $\eta$, predicted by the theory,

$$
\tau(T)=\frac{V_{\mathrm{eff}}^{*} \eta(T)}{k\left(T-T^{*}\right)^{\gamma}}
$$

reproduces the experimental data in a very good manner with the critical exponent $\gamma$ value close to the unity. $V_{\text {eff }}^{*}$ is the effective volume of the relaxing entity, and $k$ is the Boltzmann constant.

It was shown [11] that nematic state could also be reached by increasing, at constant temperature, the number of mesogenic molecules in the unit volume. The increase can be realized by growing the concentration of mesogenic molecules dissolved in nonmesogenic solvent. In analogy to the studies of the pretransitional effects in pure mesogenic compounds, we found that the effects studied in the solutions (at constant temperature) can be characterized by the virtual concentration $X^{*}$ :

$$
\tau(X)=\frac{V_{\mathrm{eff}}^{*} \eta(X)}{k T\left(X^{*}-X\right)^{\alpha}}
$$

Here $X$ is the molar fraction of the mesogenic solute, and $X^{*}$ stands for the concentration of the virtual transition of the second order, in analogy to $T^{*}$ in the Landau-de Gennes theory, and $\alpha$ is the critical exponent. It was found that for the system studied, the value $X^{*}$ is very close to 6 CHBT concentration for which the transition to homogeneous nematic phase occurs [11]. These nonlinear dielectric relaxation experiments on 6CHBT solutions were extended to the benzene solutions of the more polar $4,4^{\prime}-n$-hexylcyanobiphenyl, $\mathrm{C}_{6} \mathrm{H}_{13}-\mathrm{Ph}-\mathrm{Ph}-\mathrm{CN}$ (6CB) in this paper; the dipole moment values of the single $6 \mathrm{CHBT}$ and $6 \mathrm{CB}$ molecules are $3.5 \mathrm{D}[13]$ and $4.9 \mathrm{D}[14]$, respectively.

Recent performed nonlinear dielectric relaxation studies of solution of nonpolar molecules 4-n-butylcyclohexanecarboxylic acid, $\mathrm{C}_{4} \mathrm{H}_{9}-\mathrm{CyHx}-\mathrm{COOH}$ (BCHA) in cyclohexane [15] create opportunity for their comparison with results for the solutions of polar molecules of $6 \mathrm{CB}$. Comparative investigations of the pretransitional effects in the isotropic phase of mesogens consisting of the polar and nonpolar molecules are attractive for many reasons. First of all it should be verified whether in both cases the intermolecular interactions lead to the nematic phase 
through the pseudonematic domains, and whether are dependent on polarity of the mesogenic molecules. Besides, it should be examined whether critical-like behaviors of the nonlinear dielectric relaxation time in vicinity of isotropic-nematic phase transition could be interpreted within the same theory. The comparison of the dynamics of the nonlinear dielectric properties occurring for these two kinds of mesogenic molecules (polar and nonpolar) dissolved in nonpolar medium is the central goal of the present paper.

\section{Experimental}

$6 \mathrm{CB}$ (the transition from the nematic to isotropic phase at $T_{\mathrm{NI}}=29.8^{\circ} \mathrm{C}$ ) was synthesized and purified at the Institute of Chemistry, Military University of Technology, Warsaw. The purity of the compound, checked by the chromatography, was $99.5 \%$. Benzene was distilled and stored over molecular sieves of $4 \AA$. Figure 1 presents the phase diagram for the $6 \mathrm{CB}+$ benzene mixture.

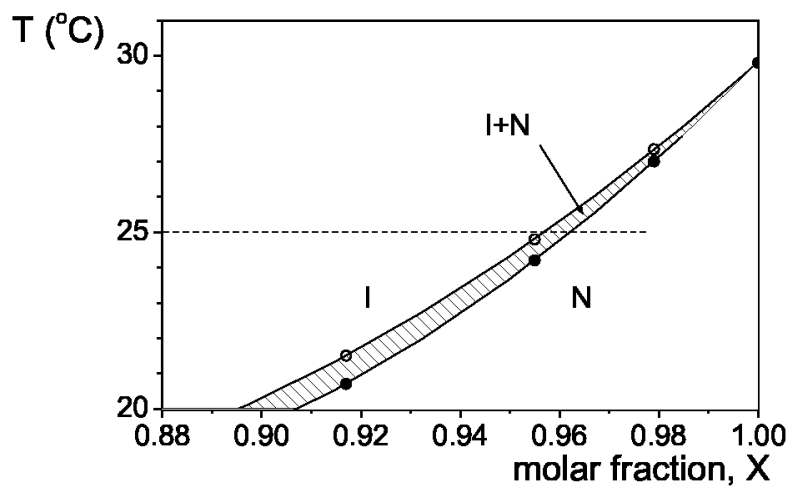

Fig. 1. Phase diagram for $6 \mathrm{CB}+$ benzene mixtures, $X$ denotes the molar fraction of $6 \mathrm{CB}$. The nonlinear dielectric spectra were recorded at $25^{\circ} \mathrm{C}$. The hatched area denotes the isotropic + nematic $(\mathrm{I}+\mathrm{N})$ two-phase region.

The nonlinear dielectric measurements were performed at $25^{\circ} \mathrm{C}$. In the method we used the electric field of high strength $E_{0}=1.1 \times 10^{7} \mathrm{~V} / \mathrm{m}$ and low frequency $(85 \mathrm{~Hz})$ perturbed periodically the system and the permittivity change

$$
\Delta \varepsilon=\varepsilon_{E_{0}}-\varepsilon
$$

was measured with a weak field $E(\omega)$ of high frequency. The nonlinear dielectric data were obtained by monitoring the modulation of the parameters of a resonant circuit induced by the application of a high field to the capacitor of the circuit, filled with the liquid studied. An LC resonance circuit with exchangeable coils covers the frequency range from $300 \mathrm{kHz}$ to $100 \mathrm{MHz}$. The details of the method and the setup used are described in $[11,16]$. 


\section{Results and discussion}

Figure 2 presents the nonlinear dielectric spectra recorded for solutions of $6 \mathrm{CB}$ in benzene, at $25^{\circ} \mathrm{C}$. The dielectric nonlinearity can be caused, in general, by two molecular phenomena occurring in isotropic liquids. First of all, the Langevin saturation of the dipoles orientation in strong electric fields. This effect leads to a decrease in the permittivity of liquids. The nonlinear dielectric increment $\Delta \varepsilon$ (4) is negative and is proportional to the square of the field strength $E_{0}$. The relaxation of the increment occurs in the same frequency region as the linear dielectric relaxation [17]. This negative effect was observed for diluted solution of 6CHBT in benzene $[17,18]$. The second main phenomenon, which can also cause a nonlinearity in the dependence of the polarization on the field strength, occurs for systems in which the intermolecular interactions (e.g., dipole-dipole or hydrogen bonds)
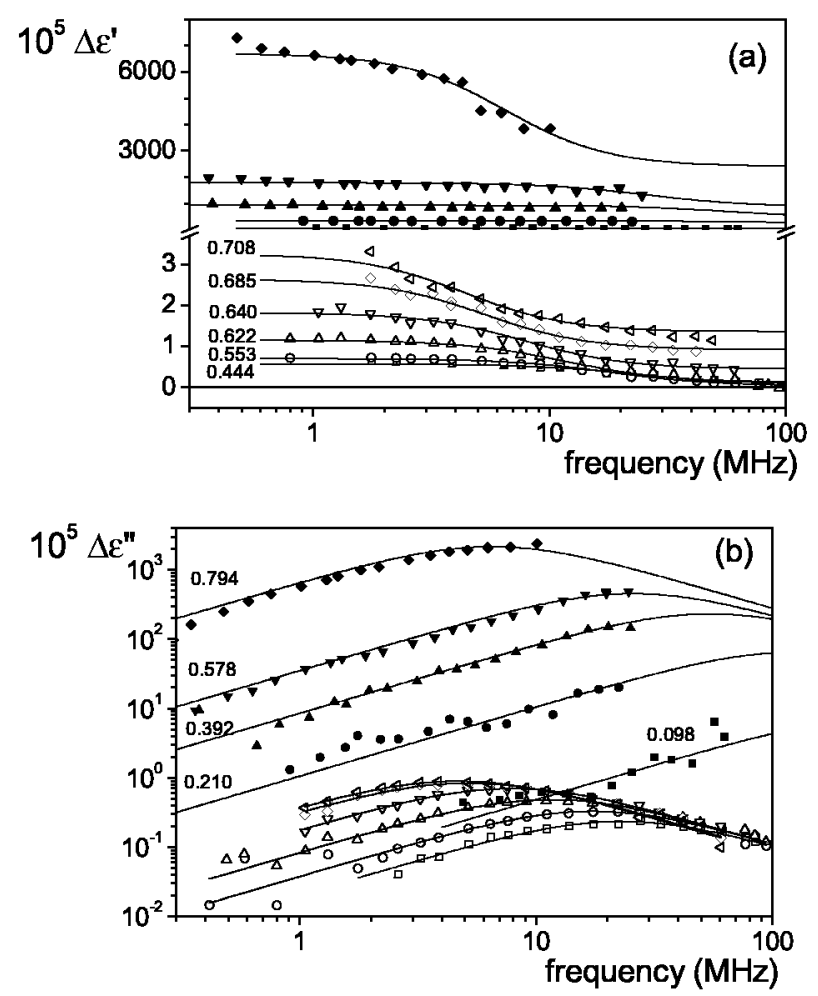

Fig. 2. Frequency dependence of the real (a) and imaginary (b) parts of the nonlinear dielectric increment measured for different molar fractions of polar $6 \mathrm{CB}$ in benzene (filled points) and nonpolar BCHA in cyclohexane (open points) [15] at $25^{\circ} \mathrm{C}, E_{0}=$ $1.1 \times 10^{7} \mathrm{~V} / \mathrm{m}$. The solid lines correspond to the Debye-type function (5) with a single relaxation time. 
lead to formation of aggregates with a compensated dipole moment. The strong electric field shifts the equilibrium between the aggregates and the monomers (or other aggregates) in favor of more polar species. This effect causes an increase in the permittivity of the liquid; i.e. the increment $\Delta \varepsilon$ (4) is positive and shows the relaxation in the frequency range corresponding to the kinetics of the aggregation process [19]. In the case of dipolar mesogenic molecules dissolved in a nonpolar medium, the positive nonlinear increment of permittivity can be related to the orientational effects, forced by the strong electric field upon the molecular aggregates - the pseudonematic domains - existing in the isotropic solutions.

The strong electric field applied to the solutions of polar 6CB induces an increase in the electric permittivity i.e. the increment (4) is positive. The positive nonlinear effect increases when the concentration of $6 \mathrm{CB}$ rises and at the same time the Langevin dielectric absorption becomes so significant that our experimental setup was no longer able to detect the nonlinear dielectric effects with a reasonable precision for the frequencies $>30 \mathrm{MHz}$. In contrary to the solutions of polar $6 \mathrm{CB}$, the Langevin dielectric absorption is no significant for solutions of nonpolar $\mathrm{BCHA}$ and the complete nonlinear dielectric absorption spectra could be recorded (Fig. 2) [15].

As shows Fig. 2, the values of the increment is above three orders of magnitude greater than measured for nonpolar BCHA. Carboxylic acids have long been known to exhibit liquid crystallinity as a result of dimerization, where the linkage of two polar molecules by two hydrogen bonds leads to the cyclic dimers with an exceptionally compensated dipole moment. The small strength of nonlinear dielectric effect (NDE) is caused by very small polarity of $\mathrm{BCHA}$ dimers.

The experimental nonlinear dielectric spectra presented in Fig. 2 can be reproduced in a very good manner by the Debye-type function with a single relaxation time $(\tau)$ :

$$
\Delta \varepsilon^{*}=\Delta \varepsilon^{\prime}-\mathrm{i} \Delta \varepsilon^{\prime \prime}=\frac{\Delta \varepsilon^{0}}{1+\mathrm{i} \omega \tau}
$$

where $\Delta \varepsilon^{0}$ is the strength of the positive nonlinear dielectric effect and $\omega$ denotes the angular frequency of the measuring electric field of low intensity. The solid lines in Fig. 2 correspond to the best fit of the function (5). The relaxation time shows a critical-like behavior as the concentration of $6 \mathrm{CB}$ approaches to the two-phase (isotropic + nematic) region (Fig. 3). As for the solutions of previously studied 6CHBT [11], one can use Eq. (3) for determination of the concentration $X^{*}$ of the virtual transition of the second order. For the concentration $X^{*}$, the viscosity to the relaxation time ratio is equal to zero, irrespective of the value of the exponent $\alpha$. As seen in Fig. 4, the dependence of $\eta / \tau$ vs. molar fraction is quite good linear and the value of $X^{*}$ obtained from the extrapolation of $\eta / \tau$ to zero, is equal to 0.96 . This value of molar fraction corresponds to the two-phase (isotropic + nematic) region of the mixture $6 \mathrm{CB}+$ benzene. Figure 5 shows that linear relation of $\ln (\eta / \tau)$ vs. $\ln \left(X^{*}-X\right)$, predicted by Eq. (3), is fulfilled in quite good manner for solutions of both, polar and nonpolar, compounds. The both slopes of the lines give for exponent $\alpha$ the same, within the experimental errors, values equal to the unity.

The fit to the Landau-de Gennes theory is good over a surprisingly wide 


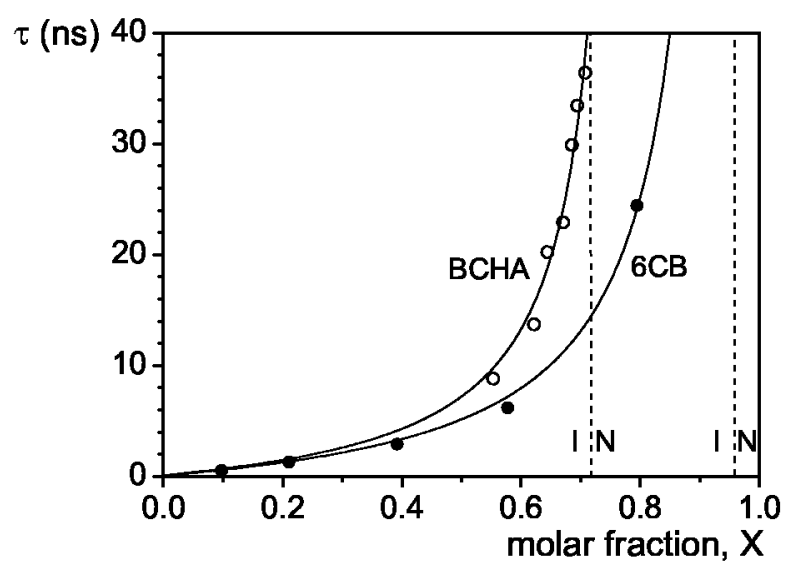

Fig. 3. Concentration dependence of the relaxation time for solutions of $6 \mathrm{CB}$ in benzene (filled circles) and $\mathrm{BCHA}$ in cyclohexane (open circles) [15] at $25^{\circ} \mathrm{C}$. The solid lines through the points are the Landau-de Gennes theoretical curves.

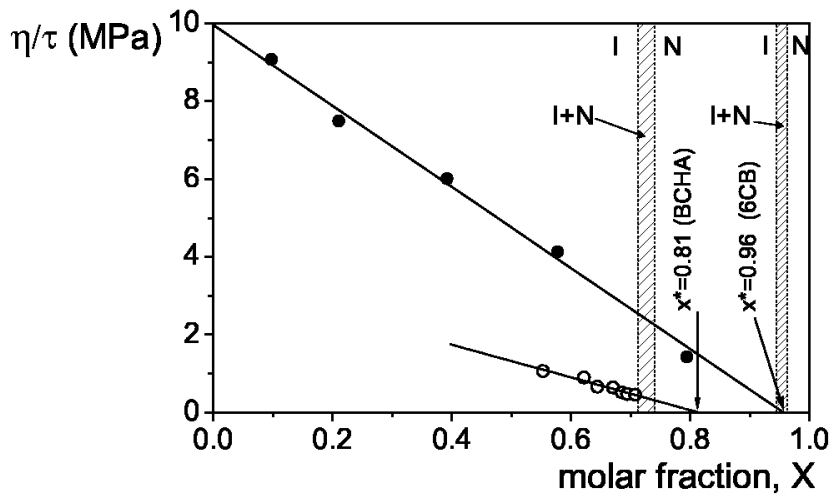

Fig. 4. Determination of the virtual molar fraction $X^{*}$ of polar $6 \mathrm{CB}+$ benzene mixture (filled circles) and nonpolar BCHA + cyclohexane mixture (open circles) [15].

concentration range of $6 \mathrm{CB}$ solutions up to the smallest $6 \mathrm{CB}$ concentration, for which the positive nonlinear increment can be detected ( 0.1 mole fraction). The correlation length, estimated similarly like in our recent paper [11], is about $\xi=3 \xi_{0}$, the value consistent with that resulting from Kerr measurements [7, 9]. For BCHA solutions the very low values of the nonlinear dielectric increment of order of $10^{-6}$ touch the limit of the sensibility of the experimental method used, and made it impossible for measurements of BCHA concentration lower than 0.55 molar fraction. The values of the fitting parameters $\left(\Delta \varepsilon^{\prime}, \tau\right)$, concentrations $X^{*}$ and critical exponents for most concentrated solutions of $6 \mathrm{CB}, 6 \mathrm{CHBT}$, and BCHA in nonpolar solvents, are gathered in Table.

The data presented in Table leads to the following conclusions: (i) according to the expectation the strength of the positive nonlinear dielectric effect strongly depends on the polarity of mesogen molecules, (ii) the values of the concentration 


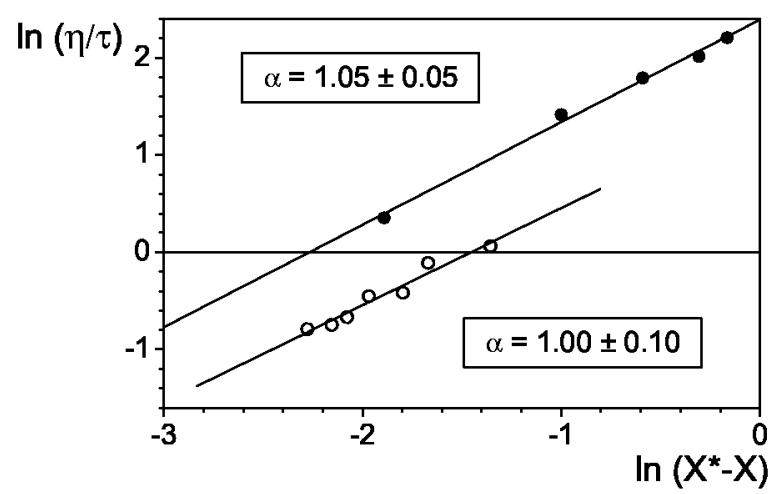

Fig. 5. Determination of the critical exponent $\alpha$ for the nonlinear relaxation time dependence on the $6 \mathrm{CB}$ concentration in benzene (filled circles) and the BCHA concentration in cyclohexane (open circles) [15] in vicinity of the transition isotropic-two-phase $(\mathrm{I}+\mathrm{N})$ regions.

TABLE

Values of the nonlinear dielectric strength, nonlinear relaxation time, concentration $X^{*}$ of the virtual transition of the second order and critical exponent $\alpha$ for solutions of $6 \mathrm{CB}, 6 \mathrm{CHBT}$, and $\mathrm{BCHA}$ in nonpolar medium at $25^{\circ} \mathrm{C}$.

\begin{tabular}{c|c|c|c|c|c}
\hline \hline Solution & $\begin{array}{c}\mu^{a} \\
{\left[10^{-30} \mathrm{C} \mathrm{m}\right]}\end{array}$ & $\Delta \varepsilon^{\prime}$ & $\tau[\mathrm{ns}]$ & $X^{*}$ & $\alpha$ \\
\hline $\begin{array}{c}6 \mathrm{CB}+\text { benzene } \\
X=0.794^{b}\end{array}$ & $16.3[14]$ & $43 \times 10^{-3}$ & 24.5 & 0.96 & $1.05 \pm 0.05$ \\
\hline $\begin{array}{c}6 \mathrm{CHBT}+\text { benzene }[11] \\
X=0.762^{b}\end{array}$ & $11.6[13]$ & $21 \times 10^{-3}$ & 18 & 0.86 & $0.92 \pm 0.13$ \\
\hline $\begin{array}{c}\text { BCHA }+ \text { cyclohexane }[15] \\
X=0.708^{b}\end{array}$ & $\approx 0$ & $19 \times 10^{-6}$ & 36 & 0.81 & $1.00 \pm 0.10$ \\
\hline
\end{tabular}

${ }^{a}$ value of the dipole moment of individual molecule;

${ }^{b}$ molar fraction of mesogenic compound

$X^{*}$ of the virtual transition of the second order are the concentration for which homogeneous nematic phase (6CHBT and $\mathrm{BCHA}$ ) or the isotropic and nematic two-phase region (6CB) occurs, and (iii) the values of the critical exponent are, within the experimental errors, close to the unity, which supports the applicability of mean field theory [1] to the solutions of these three mesogenic compounds of different polarity.

\section{Conclusions}

We have investigated the pretransitional dynamics of the solutions of mesogenic compound (6CB) in nonpolar solvent (benzene) in its isotropic phase using 
the nonlinear dielectric spectroscopy. The most remarkable observation is the identical concentration-dependent behavior of nonlinear relaxation time in vicinity of the transition to the nematic phase for solutions of polar $(6 \mathrm{CB}, 6 \mathrm{CHBT})$ and nonpolar (BCHA) mesogenic compounds. All three liquid crystals manifest the pretransitional dynamic effects, which can be interpreted within the same Landau-de Gennes theory. In particular, the critical exponent, corresponding to the nonlinear relaxation time dependence on mesogen concentration, has the same value for all three compounds. The mechanism of formation of pseudonematic domains and its conversion into nematic phase is independent of the polarity of mesogenic molecules. These observations lead to the conclusion that the critical-like behavior of the nonlinear dielectric relaxation should be common to many liquid crystals in the isotropic phase near the isotropic to nematic phase transition.

\section{Acknowledgments}

This work was supported by the Polish Research Project No. 2P03B 15822 coordinated by the Committee for Scientific Research.

\section{References}

[1] P.G. de Gennes, Physics of Liquid Crystals, Oxford University Press, Oxford 1974.

[2] J.D. Litster, T.W. Stinson III, J. Appl. Phys. 41, 996 (1970).

[3] T.W. Stinson III, J.D. Litster, Phys. Rev. Lett. 25, 503 (1970).

[4] T.D. Gierke, W.H. Flygare, J. Chem. Phys. 61, 2231 (1974).

[5] G.K.L. Wong, Y.R. Shen, Phys. Rev. Lett. 30, 895 (1973).

[6] F.W. Deeg, M.D. Fayer, J. Chem. Phys. 91, 2269 (1989).

[7] F.W. Deeg, S.R. Greenfield, J.J. Stankus, V.J. Newell, M.D. Fayer, J. Chem. Phys. 93, 3503 (1990).

[8] J.J. Stankus, R. Torre, C.D. Marshall, S.R. Greenfield, A. Sengupta, A. Tokmakoff, M.D. Fayer, Chem. Phys. Lett. 194, 213 (1992).

[9] J.J. Stankus, R. Torre, M.D. Fayer, J. Phys. Chem. 97, 9478 (1993).

[10] A. Drozd-Rzoska, S.J. Rzoska, J. Ziolo, Phys. Rev. E 54, 6452 (1996).

[11] P. Kędziora, J. Jadżyn, L. Hellemans, Phys. Rev. E 66, 021709 (2002).

[12] P. Kędziora, J. Jadżyn, L. Hellemans, Phys. Rev. E 66, 031702 (2002).

[13] P. Kędziora, J. Jadżyn, Mol. Cryst. Liq. Cryst. 192, 31 (1990).

[14] D.A. Dunmur, M.R. Manterfield, W.H. Miller, J.K. Dunleavy, Mol. Cryst. Liq. Cryst. 45, 127 (1978).

[15] P. Kędziora, J. Jadżyn, L. Hellemans, J. Phys. Chem. A, to be published.

[16] L. Hellemans, L. De Maeyer, J. Chem. Phys. 63, 3490 (1975).

[17] J. Jadżyn, P. Kędziora, L. Hellemans, Phys. Lett. A 251, 49 (1999).

[18] P. Kędziora, J. Jadżyn, L. Hellemans, Z. Naturforsch. A 54, 585 (1999).

[19] K. De Smet, P. Kędziora, J. Jadżyn, L. Hellemans, J. Phys. Chem. 100, 7662 (1996). 